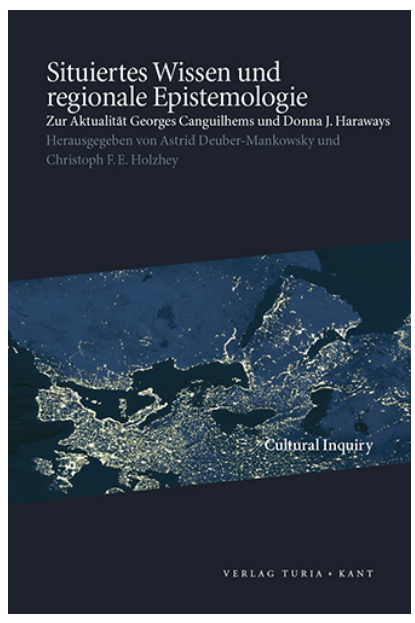

Situiertes Wissen und regionale Epistemologie: Zur Aktualität Georges Canguilhems und Donna J. Haraways, ed. by Astrid Deuber-Mankowsky and Christoph F. E. Holzhey, Cultural Inquiry, 7 (Wien: Turia + Kant, 2013), pp. 121-39

\section{CHRISTOPH F. E. HOLZHEY (i⿱ \\ Kippbilder des Vitalen \\ Lebenswissen bei Canguilhem und Haraway}

CITE AS:

Christoph F. E. Holzhey, »Kippbilder des Vitalen: Lebenswissen bei Canguilhem und Haraway $\ll$, in Situiertes Wissen und regionale Epistemologie: Zur Aktualität Georges Canguilhems und Donna J. Haraways, hg. v. Astrid Deuber-Mankowsky und Christoph F. E. Holzhey, Cultural Inquiry, 7 (Wien: Turia + Kant, 2013), S. 121-39<https: //doi.org/10.37050/ci-07_06>

RIGHTS STATEMENT:

(C) by the author(s)

This version is licensed under a Creative Commons AttributionShareAlike 4.0 International License. 


\title{
KIPPBILDER DES VITALEN
}

\author{
Lebenswissen bei Canguilhem und Haraway
}

Christoph F. E. Holzhey

In der Einleitung zu seinem Buch Die Erkenntnis des Lebens legt Georges Canguilhem besonderen Wert darauf, die Erkenntnis im Leben zu verorten. Er verwirft die Vorstellung, wonach Erkenntnis ein Ziel an sich sein könne, das vom Leben losgelöst oder ihm gar entgegengesetzt sei, wie allzu häufig angenommen wird; stattdessen bestimmt er sie durch ihre Funktion im und für das Leben. Erkenntnis ist eine Form der Auseinandersetzung mit Hindernissen oder genauer »eine allgemeine Methode zur direkten oder indirekten Auflösung der Spannungen zwischen Mensch und Milieu «. ${ }^{1}$ »Doch «, so merkt Canguilhem an, »die Erkenntnis so zu definieren heißt, ihren Sinn in ihrem Endzweck zu finden, der darin besteht, dem Menschen eine neues Gleichgewicht mit der Welt, eine neue Form und eine neue Organisation seines Lebens zu ermöglichen. ${ }^{2}$ Ein solch funktionales Verständnis von Erkenntnis hat epistemologische Folgen auch im Sinne einer Erkenntniskritik, wenngleich die Möglichkeit einer reinen Vernunft dadurch bestritten wird. Canguilhem ist der Auffassung, »dass ein vernünftiger Rationalismus seine Grenzen anerkennen und die Bedingungen seiner Ausübung einbeziehen muss «. ${ }^{3}$ Dabei scheint er hinsichtlich der Frage zu zögern, ob alle Erkenntnis dem Leben einzuschreiben sei oder ob dies nur für jene Erkenntnis gelte, die das Leben zum Gegenstand hat. Doch mit Blick auf die Lebenswissenschaften ist mit der Formulierung "Erkenntnis des Lebens « sicherlich jene Doppelbedeutung intendiert, kraft derer das Leben nicht nur als das Objekt der Erkenntnis, sondern auch als ihr Subjekt, ihre Bedingung und ihr Ursprung erscheint. Obwohl die Erkenntnis des Lebens demnach zirkelförmig verläuft, möchte ich betonen, dass es sich nicht um einen Teufelskreis handeln muss. Vielmehr lassen solche Zirkel vielfältige, in sich stimmige Lösungen zu, deren Auswahl in verschiedenen historischen, räumlichen und diskursiven Regionen letztlich kontingent ist und nur durch Beobachtung und Erfahrung festgestellt werden kann. Dementsprechend fährt Canguilhem fort: »Der Verstand darf sich auf das Leben nur beziehen, wenn er 
die Originalität des Lebens anerkennt. Das Denken des Lebendigen muss die Idee des Lebendigen dem Lebendigen selbst entnehmen. ${ }^{4}$ Und er beschließt die Einleitung mit den Worten: »Wir haben den Verdacht, dass es für die Mathematik genügen würde, Engel zu sein; um aber Biologie zu betreiben - selbst mit Hilfe des Verstandes - müssen wir uns zuweilen wie Tiere fühlen. $\aleph^{5}$

Die von mir eingangs gewählte Formulierung, dass Canguilhem die Erkenntnis im Leben verortet, soll auf Donna J. Haraways Begriff des »situierten Wissens « (situated knowledges) anspielen. Tatsächlich entspricht die Unfähigkeit der Engel zur Biologie Haraways Zurückweisung des von ihr so genannten "göttlichen Tricks« (god-trick), d. h. jenes Ideals einer objektiven Erkenntnis, die einem körperlosen Blick von nirgendwo entspräche. Doch indem ich meine Auseinandersetzung mit der Situierung des Wissens, insbesondere des biologischen Wissens, bei Canguilhem und Haraway mit Canguilhems kurzer Einleitung in Die Erkenntnis des Lebens beginne, möchte ich auf eine bestimmte Spannung aufmerksam machen, der man sich über die unterschiedliche Ausdrucksweise beider nähern kann: Während Haraway von »Wissen « im Plural spricht (knowledges), werden bei Canguilhem sowohl »Leben « als auch »Wissen « bzw. "Erkenntnis « (connaissance) im Singular verwendet. Durch die Hervorhebung dieses vielleicht nur textredaktionellen Unterschieds verfolge ich nicht etwa die Absicht, Haraway und Canguilhem auf dieser Grundlage gegenüberzustellen. Denn die Pluralisierung von Epistemologien erscheint mir als das Anliegen beider, wenn sie Erkenntnis und Wissen dadurch in ihrer Reichweite einschränken und zugleich autonom machen, dass sie diese auf historische und regionale Räume eingrenzen, die nicht nur den geographischen Raum umfassen, sondern auch diskursive und disziplinäre ebenso wie physische, körperliche Räume lebender Körper. Allerdings möchte ich einen gewissen Widerspruch bzw. ein Paradox hervorheben, was die Verfassung des Lebens im Rahmen eines solchen Projekts anbelangt: Leben erscheint als ein einziges, durchgehendes Prinzip von Pluralisierung, Komplexität und Erneuerung. Man beruft sich auf das Leben, um Kontingenz, Historizität, Fehlerhaftigkeit, Pluralität und Irreduzibilität zu betonen, doch riskiert man dadurch paradoxerweise, Leben zu etwas Notwendigem, Ahistorischem, Universellem und Einheitlichem zu substanzialisieren und dadurch die ganze Problematik des Vitalismus aufzurufen. Ich sage »riskieren ", weil ich das Paradox in dieser Form für zu einfach halte und denke, dass es einer näheren Betrachtung bedarf. Trotzdem mag 
das Paradox einer einzigen Kraft der Pluralisierung zunächst als eine Art Kürzel für eine Spannung dienen, die sowohl Kontinuität als auch Diskontinuität in Zeit und Raum bejaht; eine Spannung zwischen der Kontinuität des Lebens und der Diskontinuität, die es hervorbringt (etwa Geburt und Tod einzelner Organismen); aber auch die Spannung zwischen einer Sicht der Geschichte als einer sich unaufhörlich entwickelnden und einer von Brüchen gezeichneten; oder schließlich die Spannung zwischen der Auffassung, wonach alles im Wesentlichen von Beginn an determiniert sei, und der Behauptung der Möglichkeit wahrhaftiger Neuerung.

In seinem Essay über »Aspekte des Vitalismus « von 1947 spricht Canguilhem von einem "dialektischen Prozess « und vertritt die Auffassung, dass das Leben »die dialektische Essenz ist und dass sich das Denken dessen Strukturen zu eigen machen muss « ${ }^{6}$ Ich möchte diese Textstelle ausführlicher zitieren, da in ihr der zentrale Punkt zum Ausdruck kommt, der mich an dieser Stelle interessiert, und sie es darüber hinaus erlaubt, das Kippbild als alternative Interpretationsfigur zur Dialektik einzuführen:

Die biologische Theorie offenbart sich durch ihre Geschichte hindurch als ein geteiltes und oszillierendes Denken. Mechanismus und Vitalismus stehen sich im Hinblick auf das Problem der Strukturen und Funktionen gegenüber; Diskontinuität und Kontinuität im Hinblick auf die Abfolge der Formen; Präformation und Epigenese im Hinblick auf die Entwicklung des Seins; Atomizität und Ganzheit im Hinblick auf das Problem der Individualität.

Dieses permanente Oszillieren, das Zurückkehren zu Positionen, von denen sich das Denken scheinbar definitiv verabschiedet hatte, kann unterschiedlich interpretiert werden. In einer Hinsicht kann man sich fragen, ob es wirklich einen theoretischen Fortschritt gibt - abgesehen von der Entdeckung neuer experimenteller Tatsachen [...]. In einer anderen Hinsicht kann man dieses augenscheinliche theoretische Oszillieren aber auch als Ausdruck einer verkannten Dialektik betrachten, insofern die Rückkehr zur selben Position nur eine optische Täuschung ist, die darin besteht, dass man einen Punkt im Raum, der sich auf der Vertikalen ständig verschiebt, mit einer identischen Position auf derselben Ebene verwechselt. Überträgt man den dialektischen Prozess des Denkens in die Wirklichkeit, kann man behaupten, dass der Forschungsgegenstand selbst, das Leben, die dialektische Essenz ist und dass sich das Denken dessen Strukturen zu eigen machen muss. Das Leben geht über die Gegensätze von Mechanismus und Vitalismus, Präformation und Epigenese hinaus und setzt sich bis in die Theorie des Lebens hinein fort. 
Die Vitalität des Vitalismus verstehen heißt, sich auf eine Unterscheidung der Sinnzusammenhänge zwischen dem Leben und der Wissenschaft im Allgemeinen, dem Leben und der Wissenschaft vom Leben im Besonderen einzulassen. ${ }^{7}$

Das Bemerkenswerteste an diesen Ausführungen ist die Feststellung eines »permanenten Oszillierens «. Dies widerspricht scheinbar, wie ich weiter unten ausführen werde, dem Geist der historischen Epistemologie. Während die zweite angebotene Deutung - eine »verkannte Dialektik «, die in die Wirklichkeit übertragen werden könnte - die Dinge zu verschlimmern scheint, indem sie das Leben als »dialektische Essenz « in einer transhistorischen Ontologie festlegt, möchte ich vielmehr eine Variation der ersten Deutung, die jeden wirklichen theoretischen Fortschritt in Frage stellt, erkunden.

Um genauer zu sein: Ich möchte die Frage stellen, was es bedeuten könnte, das Leben - oder das Lebendige und damit auch die Lebenswissenschaften - als etwas zu betrachten, das als ein Kippbild konstituiert wird, welches zwischen verschiedenen Aspekten ohne Vermittlung oder Synthese oszilliert und welches seine Einheit allein in den Phänomenen des Lebens und in einem Namen findet, nicht aber in einem Begriff oder einer Theorie innerhalb einer logischen Ordnung. Ein berühmtes Beispiel einer solchen Kippfigur ist das Hasen-Enten-Bild, das Ludwig Wittgenstein in seinen Philosophischen Untersuchungen anführt. Das Bild kann als das einer Ente oder eines Hasen angesehen werden; die beiden Aussagen »Es ist eine Ente « - Es ist ein Hase « widersprechen sich scheinbar und die Feststellung, es sei beides zugleich, scheint paradox zu sein. Wir können uns problemlos zwischen diesen beiden Ansichten hin- und her bewegen, aber der Widerspruch ist anscheinend unlösbar; keine Vermittlung, vor allem kein dialektischer Fortschritt scheint hier möglich. Zugleich ist aber auch nichts Mysteriöses an dem Gegenstand in seiner materiellen oder formalen Verfassung. Wir könnten sagen, dass der Widerspruch von einer Hinzufügung des erkennenden Geistes herrührt, der eine Strichzeichnung in eine wahrgenommene und wiedererkannte Gestalt verwandelt - ein Vorgang, der zu unserer Orientierung nützlich sowie für unser Überleben notwendig ist und üblicherweise ohne Widerspruch vonstattengeht, der aber letztlich ein Schein ist. Während es wohl zu weit ginge, von einem notwendigen Schein zu sprechen - Wittgenstein zieht auch die Möglichkeit einer Aspektblindheit in Betracht -, gibt es dennoch einige Anhaltspunkte, um dieses Phänomen mit Kants transzendentalem Schein, und seinen 
Antinomien in Beziehung zu setzen, insbesondere auch der Antinomie der teleologischen Urteilskraft, welche die Betrachtung des Lebenden sowohl unter dem Aspekt der mechanischen Verursachung als auch dem der Zweckmäßigkeit betrifft. ${ }^{8}$

Zweifellos ist das Kippbild-Modell zu einfach, um umstandslos übertragen werden zu können, zumal im Falle des Lebens - wie in allen interessanten Fällen - das Objekt selbst zur Debatte steht. Im Gegensatz zum Hasen-Enten-Bild ist es in der Tat im Allgemeinen unklar, ob widerstreitende Perspektiven auf ein Phänomen als unterschiedliche Perspektiven auf ein und denselben Gegenstand erachtet werden können. Der Grund, weshalb ich das Kippbild-Modell an dieser Stelle bemühe, besteht vielmehr darin, dass es die Möglichkeit veranschaulicht, dass widerstreitende Perspektiven lediglich unterschiedliche Aspekte desselben Gegenstands erfassen, wodurch eine alternative Denkfigur angeboten wird gegenüber dem Drang, entweder eine Perspektive auszuschließen oder die Synthese verschiedener Perspektiven anzustreben. ${ }^{9}$ Zum zweiten bin ich daran interessiert zu erkunden, was es bedeutet, dass »das Denken « allem Anschein nach darauf beharrt, manche seiner Gegenstände als Kippbilder zu konstituieren. Auch wenn dies zugleich eine Frage von Logik und Epistemologie ist, geht es mir darum herauszufinden, ob es sich letztendlich nicht um eine Frage der Ästhetik oder des Begehrens seitens lebendiger, menschlicher Subjekte der Erkenntnis handelt. Während schließlich die Aufspaltung der Perspektiven auf ein vermeintlich selbes Objekt eine Konstante darstellen mag, gibt es doch generell eine Vielfalt spezifischer Perspektiven mit unterschiedlichen Implikationen und Folgen, was die Wahl einer bestimmten Perspektive zu einer ethischen oder politischen Frage macht.

Sicherlich wird es mir nicht möglich sein, all dies im Rahmen dieses Essays detailliert zu behandeln, doch will ich versuchen, das Potential des Kippbild-Modells für das Nachdenken darüber anzuzeigen, wie das Leben als ein Objekt des Denkens mobilisiert und dazu gemacht wird. Beginnen möchte ich mit der Problematik des Vitalismus, den Canguilhem in der zitierten Passage auf mehrdeutige Weise, gewissermaßen durch eine Verdoppelung, verteidigt: Einerseits ist der Vitalismus innerhalb eines permanent oszillierenden und möglicherweise dialektischen Prozesses nur eine Seite eines möglichen Gegensatzpaars, doch anderseits ist die Synthese dieses Prozesses im Leben oder eben in der »Vitalität des Vitalismus « zu finden. 
Auch wenn Canguilhem den Vitalismus auf eine offene, wenngleich kritische und selbstreflexive Weise gutheißt, ist die Rolle des Vitalismus in seinem Denken und Werk umstritten. ${ }^{10}$ Er ist sich sicherlich dessen bewusst, dass der Vitalismus in überwiegendem Maße als eine unhaltbare Perspektive in den Lebenswissenschaften des 20. Jahrhunderts angesehen wird, und genau aus diesem Grunde lässt er sich darauf ein. Er weist darauf hin, dass der schlechte Ruf des Vitalismus unter Biologen davon herrührt, dass er nicht klar genug vom »Animismus « unterschieden wird und dass er mit metaphysischen Theorien über die Essenz des Lebens assoziiert wird. Die Vitalisten des 18. Jahrhunderts seien tatsächlich vorsichtige Positivisten, d. h. Newtonianer, gewesen. Sie würden sich auf eine vitale Kraft berufen, jedoch nicht zum Zweck einer wesentlich tautologischen - Erklärung, sondern um die irreduzible Besonderheit des Lebens und des biologischen Wissens anzuerkennen. ${ }^{11}$ So verstanden, stellte der Vitalismus eher Fragen, als dass er Antworten lieferte. Canguilhem betont, dass er in seiner Verteidigung des Vitalismus keinesfalls behaupte, im Besitz eines vitalistischen Schlüssels zu den Problemen zu sein, die das Leben an unseren Verstand stellt. So beteuert er, »dass wir nicht an die geheimnisvollen Türen des Lebens glauben«, und fügt dem hinzu: »Man kann annehmen, dass das Leben die Logik aus der Fassung bringt, ohne gleichzeitig zu glauben, man würde besser mit ihm zurechtkommen, wenn man auf die Bildung von Begriffen verzichtet, um irgendeinen verlegten Schlüssel zu finden. «12 Demnach bezeichnet der Vitalismus ein Erfordernis; er ist »eher ein Anspruch als eine Methode und vielleicht eher eine Moral als eine Theorie $"{ }^{13}$ Diese Passage zitiert Michel Foucault in seiner Einleitung zur englischen Ausgabe von Canguilhems Das Normale und das Pathologische, wo er vom Vitalismus als »kritischem Indikator « spricht. ${ }^{14}$ Im Gegensatz zu einem metaphysischen Vitalismus etwa nach Art der deutschen Lebensphilosophie zu Beginn des 20. Jahrhunderts birgt diese Art von Vitalismus weiterhin ein kritisches Potential in sich, wie Astrid Deuber-Mankowsky und ich im Bezug auf Donna J. Haraways Werk unlängst vertreten haben. ${ }^{15}$

Jedoch stellt die Profilierung einer kritischen Form von Vitalismus eine Herausforderung dar, zumal Canguilhems Konzeption des Vitalismus letztlich mehrdeutig bleibt - und dies vielleicht aus gutem Grund. So stellt Monica Greco in einer neueren Untersuchung mit dem Titel »On the Vitality of Vitalism« heraus: 
[I]t is possible to misunderstand Canguilhem in more than one way; that is, not only in the naive sense of failing to see the difference between his vitalism and classical forms of the idea, but also in the sense of failing to see a certain continuity between them, by underplaying the reference to the ontology of life and underestimating its significance. ${ }^{16}$

Entgegen der weitverbreiteten Auffassung, dass Canguilhems Vitalismus obsolet und letztlich befremdlich sei oder aber seiner ontologischen Implikationen entledigt werden könne, versucht Greco ein »homologue to Canguilhem's vitalism in contemporary forms of scientific thought " $\mathrm{zu}$ identifizieren. ${ }^{17}$ Grecos aufschlussreicher Artikel ist im Kontext des vorliegenden Bandes insofern besonders hilfreich, als sie im Zuge der angestrebten Aktualisierung von Canguilhems Vitalismus ausdrücklich auf Donna J. Haraway zu sprechen kommt. Interessanterweise geschieht dies nicht im Kontext des Vitalismus, sondern von Individualität und Anthropozentrismus. Doch indem Greco eine systemtheoretische, organismuszentrierte Sicht des Lebens und die Komplexitätstheorie als mögliche Homologien zu Canguilhems Vitalismus vorführt, zeigt sie implizit weitere Verknüpfungen auf.

Nach ihrer Doktorarbeit Crystals, Fabrics, and Fields, die sie 1976 veröffentlichte, scheint sich Haraway tatsächlicher eher wenig zum Vitalismus zu äußern. Sie legt dort ihren Schwerpunkt auf die Beiträge von Ross G. Harrison, Joseph Needham und Paul Weiss zur Entwicklung des Organizismus in der ersten Hälfte des 20. Jahrhunderts als eines Paradigmas, das den jahrhundertealten Gegensatz zwischen Vitalismus und Mechanismus überschreitet. In ihrer Einleitung zur Neuauflage von 2004 nimmt Haraway erneut die Polemik des Buchs auf, wobei sie darauf hinweist, dass sich ihre Strategie mittlerweile geändert habe nach 30 Jahren feministischer Kritik an dem klassischen, teleologischen Narrativ, das sie ursprünglich verfolgte, und sie nun wie folgt charakterisiert: »A hero - materialist organic systems theory - defeats the dragons of mechanism and vitalism. ${ }^{18}$ Während sie nun nach anderen Narrationsweisen und Erklärungswegen suche, bleibe das Bestreben, einen dritten Weg zum Vitalismus-Mechanismus-Gegensatz zu finden, auch weiterhin bestehen: »If I tried to evade the misleading alternatives of mechanism and vitalism in this book [...], in my work today I try to find a third way oblique to the geometries of wholes, fragments, and central axes. ${ }^{19}$

Distanziert sich Haraway vom Vitalismus, wohingegen Canguilhem ihn verteidigt, so scheint dies weitestgehend terminologische Gründe zu 
haben. Anders als Canguilhem sieht Haraway Vitalisten aller Couleur von einer nicht-physikalischen Substanz ausgehen, während Organizisten ohne eine solche Annahme zurechtkämen. ${ }^{20}$ Demgegenüber möchte ich nicht nur darauf hinweisen, dass der Organizismus selbst als eine Form von Vitalismus verstanden werden kann (wie wir etwa bei Greco sehen können, die sich gerade auf Paul Weiss bezieht), sondern auch nahelegen, dass schon die Suche nach einem dritten Weg zu gegensätzlichen Theorien gut mit einer vitalistischen Haltung übereinstimmt, die dabei Vitalismus eher als Erfordernis denn als Theorie begreift.

Stellte Haraways Dissertation den Organizismus zunächst als eine erfolgreiche Synthese der jahrhundertealten Dichotomie zwischen Mechanismus und Vitalismus dar, ${ }^{21}$ so verdeutlichen ihre letzten Stellungnahmen, dass es dieser Synthese nicht gelingt, alle Perspektiven auf das Lebendige zusammenzuführen. Vielmehr ergibt sich eine neue Dichotomie, nämlich diejenige zwischen Organizismus und Reduktionismus. Haraway setzt sich nun detailliert mit der Möglichkeit auseinander, dass hier eine Kontinuität vorliegt oder, mit den Worten von Hilde Heins Artikel, eine »Endurance of the Mechanism-Vitalism Controversy«. Haraway räumt zwar ein: »Hein is correct in insisting strongly that essential elements of a very traditional dispute are retained in contemporary biology «, doch hebt sie zugleich hervor: »[T]here is a crucial discontinuity between vitalism-mechanism and organicismreductionism. «22 In der Tat ist der Nachweis eines epistemologischen Bruchs - oder eines Paradigmenwechsels, wie sie in Anlehnung an Thomas Kuhn schreibt - ein zentrales Anliegen ihres Buchs. Mit Blick auf die Doppelbedeutung, die der Erkenntnis des Lebens bei Canguilhem zukommt, möchte ich hier fragen, was es bedeuten würde, solch ein Investment heraufkommender Paradigmen und epistemologischer Brüche als eine Form von Vitalismus zu lesen. Im vorliegenden Falle würde dies bedeuten, dass die Schilderung dessen, wie der Mechanismus-Vitalismus-Gegensatz an sein Ende gelangt und einer qualitativ neuen, autonomen Konstellation wie etwa dem Organizismus-ReduktionismusGegensatz gewichen sei, als eine Bestätigung des Vitalismus gelesen werden könnte. Und dasselbe würde auch für Haraways anschließende Suche nach einem drittem Wege gelten, der seinerseits über den Organizismus hinausgeht, wie sie in ihrer Einleitung von $2003 \mathrm{zu}$ verstehen gibt: 
For example, I try to ask what emergences are like without hierarchically organized levels. From heterogeneous genomes to ecological entanglements, the congeries of historical relatings that organisms are requires more venturesome geometries and topologies than organic systems theories can offer. ${ }^{23}$

Das vermeintliche Paradox infolge des Auftretens des Vitalismus an zwei verschiedenen Stellen - oder vielmehr drei Stellen, wenn man auch den Organizismus als eine Form von Vitalismus berücksichtigt, der vitalistisch aus einem Gegensatz von Vitalismus und Mechanismus hervorgeht - kann durch die nähere Bestimmung aufgelöst werden, dass es sich um einen Vitalismus qua Erfordernis handelt, der einen Vitalismus qua Theorie herausfordert und auf die Entstehung neuer Theorien und Paradigmen hin drängt. Doch trotz dieser Präzisierung, mit der ich zu Canguilhem zurückkehre, scheinen alle Versuche, zu einer befriedigenden, kritischen Konzeption von Vitalismus zu gelangen, ambivalent zu bleiben und zu neuen Vieldeutigkeiten zu führen. Es ließe sich vielleicht aus allgemeinen Gründen argumentieren, dass der Versuch, einen kritischen Vitalismus zu isolieren - als Frage statt als Antwort, als moralischer Anspruch statt als Theorie -, sich gerade insofern als instabil erweist, als dadurch die Fixierung des Vitalismus angestrebt wird. Mit anderen Worten stellt sich die Frage, ob der Vitalismus als kritischer Indikator nicht auch eine Methode definiert und ob er nicht eine Theorie und eine Antwort zumindest impliziert.

In der Tat ist es fraglich, ob Canguilhem auf vitalistische Theorien zugunsten eines puren Vitalismus des Erfordernisses verzichten könnte oder würde, denn dies würde bedeuten, die gesamte wissenschaftliche Aktivität und den gesamten wissenschaftlichen Fortschritt der anderen, mechanistischen, Seite zu überlassen. Genau darauf spielt Foucault an, wenn er hervorhebt, dass sich »in der Geschichte der Wissenschaften vom Leben ein paradoxer Sachverhalt « beobachten lasse: Ihre » Verwissenschaftlichung $[\ldots]$ konnte [...] sich doch nur in dem Maße entfalten, in dem sie unablässig das Problem der Spezifizität des Lebens [...] als Herausforderung immer wieder aufgegriffen hat «. Dies impliziere jedoch nicht, so Foucault weiter, dass der Vitalismus wahr sei, sondern nur, dass er als theoretischer und kritischer Indikator funktioniert habe und weiterhin funktioniere. ${ }^{24}$ Indes betont Canguilhem in seinem Buch über den Reflexbegriff gerade die Fruchtbarkeit vitalistischer Forschung, aus der neue Begriffe hervorgehen können, auch wenn diese letztlich eine andere, mechanistische Theorie bestätigen. ${ }^{25}$ Wenn über- 
haupt, so würde man eher vermuten, dass mechanistische Theorien verzichtbar wären, doch scheint es mir eher so zu sein, dass Canguilhem durch die Behauptung eines permanenten Oszillierens auch eine wiederkehrende Spaltung zwischen unterschiedlichen Theorien oder Zugängen behauptet und nicht lediglich zwischen der Erfordernis des Vitalismus und einem kumulativ und asymptotisch fortschreitenden Wissens. Mit anderen Worten, der Vitalismus als Erfordernis ist ein kritischer Indikator für zu vermeidende Reduktionen, von welcher Theorie sie auch bewirkt sein mögen - ob von mechanistischen oder vitalistischen, organizistischen oder reduktionistischen -, aber er stellt deren provisorischen Nutzen und Legitimität nicht in Frage: Das Leben mag die Logik aus der Fassung bringen, aber dies bedeutet nicht, dass man mit einem Verzicht auf Begriffsbildung besser auskäme.

Auch wenn Canguilhem im Weiteren die Möglichkeiten eines dialektischen Prozesses in Erwägung zieht, stellt er zugleich fest, dass es der dialektische Prozess zumindest in einer gewissen Hinsicht nicht schafft, die Spaltung zu überwinden, die sich in Anbetracht der Rekonfigurierung gegensätzlicher Begriff unablässig selbst reproduziert. Allein das Leben selbst transzendiert den Gegensatz. Als solches - d. i. als dialektische Essenz - stellt es die Begrenztheit jeder Seite heraus und verlangt nach einer Synthese; und doch führt jeder Versuch einer Synthese, d. h. der Einverleibung unterschiedlicher Aspekte eines jeden Zugangs, unweigerlich zu weiteren Spaltungen. Der Organizismus - in Haraways Dissertation als erfolgreiche Synthese dargestellt sowie in der Systemtheorie und modernen Selbstorganisations- und Emergenztheorien weiterentwickelt - mag hierfür als Beispiel dienen: Sofern bei der Emergenz ein qualitativer, objektiver Sprung, eine Diskontinuität, ein Bruch oder Schnitt betont wird, befinden wir uns meiner Auffassung nach auf der Seite eines neuen Vitalismus. Doch sofern die Betonung auf Kontinuität oder einem nur graduellen Unterschied liegt, befinden wir uns auf der Seite eines neuen Mechanismus oder kausalen Determinismus.

Worauf ich hinauswill, ist die These, dass der Vitalismus als kritischer Indikator bzw. der Begriff des Lebens als eine »dialektische Essenz « das Leben als striktes Kippbild konstituieren. Das Leben hat keine geheimnisvollen Türen, zu denen wir einen Schlüssel finden könnten. Vielmehr ist es solcherart verfasst, dass es die Logik aus der Fassung bringt und (mindestens) unter einem zweifachen Aspekt betrachtet werden muss, von denen ein jeder seinerseits eine Reduktion darstellt, woran zwar nachgebessert werden kann, was aber dessen ungeachtet 
niemals auf eine Synthese hinauslaufen wird, die es unterlässt, der Logik zu trotzen.

Insofern sie sich auf eine unbestimmte Zukunft bezieht, handelt es sich hier sicherlich um eine starke und problematische Behauptung und ist als solche auch keine Frage mehr, sondern eine bestimmte Antwort -, doch was in den Debatten über den Vitalismus im weitesten Sinne auf dem Spiel steht, ist eben genau die Frage, in welchem Maße Vitalismus überhaupt »etwas anderes ist als die Verweigerung des vom Mechanismus zur Vollendung seines Werks verlangten Aufschubs «, wie Canguilhem in »Aspekte des Vitalismus « schreibt. ${ }^{26}$ Entgegen dieser Ansicht kritisiert er den klassischen Vitalismus für die Beschränkung seines Gültigkeitsbereich auf das, was der Mechanismus nicht erklären kann, anstatt »die Originalität der Biologie [...] über die Gesamtheit der Erfahrung « zur Geltung zu bringen und »die Materie innerhalb des Lebens und die Wissenschaft der Materie, das heißt die Wissenschaften schlechthin, innerhalb der Aktivität des Lebens [zu] ১begreifen « ${ }^{27}$ Canguilhem scheint zudem einen emphatischen Begriff von Vitalismus im Sinne einer »Spontaneität « oder »Schöpfung « des Lebens zu vertreten, ${ }^{28}$ was zwar keine Theorie sein mag, die etwas erklärt, aber dennoch sicherstellt, dass der Vitalismus immer wieder als Erfordernis wiederkehren wird, wie erfolgreich die Wissenschaft in der Erklärung von Phänomenen des Lebens auch immer sein mag.

Es sei denn, natürlich, es gibt kein Leben und keine Geschichte. Canguilhems Beobachtung eines permanenten Oszillierens und »Zurückkehren[s] zu Positionen, von denen sich das Denken scheinbar definitiv verabschiedet hatte «, ist umso bemerkenswerter, als er andernorts die Suche nach Vorläufern in der Wissenschaftsgeschichte heftig kritisiert. In seinen Überlegungen zum »Gegenstand der Wissenschaftsgeschichte « von 1966 betont er nachdrücklich, dass der Gegenstand der Geschichte nicht mit dem Gegenstand der Wissenschaft selbst verwechselt werden dürfe und dass er vielmehr die Geschichtlichkeit des wissenschaftlichen Diskurses sei. In diesem Zusammenhang lehnt er die Suche nach Vorläufern ab: »Genau genommen würde die Wissenschaftsgeschichte jeden Sinn verlieren, wenn es Vorläufer gäbe, weil die Wissenschaft dann nur scheinbar eine historische Dimension hätte. ${ }^{29}$ Müssen wir nun hieraus schließen, dass angesichts der wiederholten Wiederkehr des Vitalismus die Geschichte der Lebenswissenschaften jeglichen Sinn verliert? Obwohl diese Möglichkeit mit Canguilhems Infragestellung jedes theoretischen Fortschritts in der biologischen Theorie überein- 
stimmt, liegt nach meinem Dafürhalten das Gegenteil vor, und zwar insofern, als der Vitalismus als Erfordernis - und das Leben als Quelle für dessen Vitalität - im Kern von Canguilhems Verständnis von Geschichtlichkeit liegt.

Wenn der Vorläufer jemand ist, »der einen Weg schon ein Stück weit zurückgelegt hatte, den erst kürzlich ein anderer zu Ende gegangen ist «, ${ }^{30}$ dann gibt es sicherlich Entwicklung und Wandel. Doch erscheint dieses Fortschreiten dann als ein prädeterminiertes, gleich einem von mechanistischer Kausalität bewirkten Wandel. Es scheint so, als ob für Canguilhem - und er steht hier sicherlich nicht allein - Geschichtlichkeit »genuinen Wandel « oder Neuerung erfordert, eine spontane Transformation, die nicht prädeterminiert ist und von einer solchen epistemologischen Natur ist, dass »Begriffe, Diskurse und Denkstile « nicht »von einem intellektuellen Umfeld in ein anderes beliebig versetzt werden « können. ${ }^{31}$ Die Wiederkehr des Vitalismus ist daher nicht als Wiederkehr eines Vorläufers zu verstehen, sondern im Sinne einer Manifestierung des Lebens, das sich Reduktionen widersetzt und zum Aufkommen neuer Begriffe und regionaler Epistemologien führt, ohne die es keine Geschichte im Sinne Canguilhems gäbe. So scheint schließlich in der Geschichte der Lebenswissenschaften - und nur dort - der Gegenstand der Geschichte mit dem der Wissenschaft zusammenzufallen, nämlich der Geschichtlichkeit bzw. dem Leben als genuinem Wandel.

Interessanterweise schließt Canguilhem nicht gänzlich aus, dass eine Synthese gefunden werden könnte, die dem wiederkehrenden Oszillieren Einhalt gebieten würde. In seinem Artikel zur Zelltheorie führt er die Multistabilität des Lichts an, d. h. die Möglichkeit, Licht als Teilchen oder als Welle anzusehen. Dieses in der Geschichte der Physik paradigmatische Kippbild dient ihm als Beispiel nicht nur für die Wiederkehr eines einst für obsolet erachteten Gedankens, nämlich Newtons Teilchenbild, das von Christian Huygens' und Augustin-Jean Fresnels Wellentheorie für eine Weile in den Hintergrund gedrängt wurde; sondern er spricht auch von einer »Synthese « der Teilchen- und Wellentheorie in der Quanten-Wellen-Mechanik. Zwar hält er fest, dass eine solche Synthese »die Annahme [verbietet], eine der beiden Repräsentationen des Lichtphänomens wäre zugunsten der anderen abgeschafft worden «, ${ }^{32}$ aber ebenso gut könnte man sagen, dass wir dank der Synthese letztlich ohne die beiden Repräsentationen auskommen können (ausgenommen für pragmatische Zwecke). Canguilhem kommt auf dieses Beispiel gegen Ende seines Artikels zurück, wo er schreibt: 
Der Gedanke ist keineswegs abwegig, dass sich die Biologie bezüglich der Struktur der Organismen auf dem Weg zu einer Vereinigung von Repräsentationen und Prinzipien befindet, analog zu jener, die die Wellenmechanik zwischen den beiden einander scheinbar wiedersprechenden Konzeptionen von Welle und Teilchen bewerkstelligt hat. Die Zelle und das Plasmid sind eine der beiden letzten Inkarnationen der zwei intellektuellen Bedürfnisse nach Diskontinuität und Kontinuität, die im Laufe der theoretischen Aufklärung einander unablässig gegenüberstehen - jener Aufklärung, die im Gange ist, seit Menschen denken können. ${ }^{33}$

Ein weiterer wiederkehrender Gegensatz (der von Diskontinuität und Kontinuität) stellt hier den unmittelbaren Kontext dar und dennoch ist auch hier der Vitalismus weiterhin präsent. So sieht Canguilhem im Begriff des Plasmas, dem sich Biologen immer wieder zuwenden, um den wahrgenommenen Unzulänglichkeiten der Korpuskeltheorie zu begegnen, einen »Avatar des mythologischen, alles Leben erzeugenden Fluidums, der schäumenden Woge, aus der Venus emporstieg «. ${ }^{34}$ An dieser Stelle geht es ihm nicht etwa um die Gleichsetzung von Wissenschaft und Mythologie, sondern vielmehr darum, gewisse Möglichkeiten zu eröffnen, indem aufgezeigt wird, dass die Zelltheorie ihre Grenzen hat und dass Wissenschaftler sich dessen bewusster waren, als es neuere Lehrmeinungen glauben machen. So erklärt Canguilhem am Ende seines Essays, dass

der aktuelle Bedarf an einer flexibleren und umfassenderen Theorie nur jene Geister überraschen [kann], die außerstande sind, in der Wissenschaftsgeschichte den Sinn für unterschiedliche theoretische Möglichkeiten aufzuspüren, die von denen abweichen, mit denen die Lehre der ausschließlich letzten Ergebnisse des Wissens sie vertraut gemacht hat - einen Sinn, ohne den es weder eine wissenschaftliche Kritik noch eine Zukunft der Wissenschaft gibt. ${ }^{35}$

Dieser Möglichkeitssinn, den Canguilhem in seinem Artikel als die zentrale Leistung der Wissenschaftsgeschichte erachtet, zielt auf weiteren wissenschaftlichen Fortschritt ab. Doch ist damit wirklich jene Synthese angestrebt, die nach seinem Dafürhalten die Quantenmechanik für das Teilchen-Wellen-Problem angeboten hat? Ich habe eher den Eindruck, dass das zukünftige Leben der Wissenschaft dadurch sichergestellt wird, dass das Leben als striktes Kippbild aufrechterhalten wird. In Canguilhems Artikel über die Zelltheorie scheint es in der Tat insofern eine zweifache vitalistische (oder zumindest vitale) Erfordernis zu geben, als Canguilhem hier das Thema der Individualität in den Vordergrund hebt, 
dessen Einheit nicht weniger durch Kontinuität als durch Diskontinuität gefährdet ist: Denn zum einen muss das Individuum seine kontinuierliche Einheit gegenüber den Zellen behaupten, aus denen es sich zusammensetzt, zum anderen muss es seine Autonomie mit Blick auf die Umwelt wahren, deren Teil es ist.

Wie bereits erwähnt, argumentiert Donna J. Haraway in ihrer Dissertation zunächst, dass der Organizismus eine erfolgreiche Synthese von Vitalismus und Mechanismus bildete, erkennt aber dann die Wiederkehr einer Spaltung an - dieses Mal zwischen Organizismus und Reduktionismus - und betont dabei, dass sich dennoch ein Paradigmenwechsel vollzogen habe. Um mit Canguilhem zu sprechen, könnte man sagen, dass sie den Eindruck eines nicht stattfindenden theoretischen Fortschritts auf eine optische Täuschung zurückführt - nämlich auf eine Sichtweise, die dieselben binären Gegensätze in der Geschichte wiederkehren sieht - und dass sie damit ihrerseits die Präsenz genuiner Geschichtlichkeit betont. Während sich ihre Dissertation mit der durch den Organizismus erreichten Synthese einigermaßen einverstanden zeigte, lässt sich behaupten, dass ihre späteren Arbeiten demgegenüber fortwährend bestrebt sind, Emergenzen in Raum und Zeit zu erneuern und so zu einer Vielfalt epistemologischer Regionen zu gelangen, die um nochmals aus ihrer Einleitung von 2003 zu zitieren - »without hierarchically organized levels « und mit "more venturesome geometries and topologies than organic system theories can offer " hervorgehen. ${ }^{36}$

Wenn Canguilhem das Leben als dialektische Essenz begreift, welche wiederkehrende Gegensätze transzendiert, und ich vorgeschlagen habe, dies als Konzeption des Lebens als ein Kippbild zu lesen, dann scheint Haraway etwas Vielfältigeres und Mobileres anzuvisieren. Allerdings passt etwa ihre Verwischung des Unterschieds von Maschine und Organismus sehr gut zu Canguilhems Sicht der Technik, ${ }^{37}$ und sie fährt fort, nicht nur das proto-vitalistische Paradigma der Emergenz zu mobilisieren, sondern auch Gegensätze zu errichten und gleichzeitig zu hinterfragen. Im Zuge dessen stellt sie auch zahlreiche Kippbilder her. So wird beispielsweise ihr Cyborg-Mythos, der »von überschrittenen Grenzen, machtvollen Verschmelzungen und gefährlichen Möglichkeiten « ${ }^{38}$ handelt und der den in den 80er Jahre beherrschenden OrganismusTechnologie-Dualismus anficht, zu einem Kippbild:

Aus einer Perspektive könnte das Cyborguniversum dem Planeten ein endgültiges Koordinatensystem der Kontrolle aufzwingen, die endgültige Abs- 
traktion, verkörpert in der Apokalypse des im Namen der Verteidigung geführten Kriegs der Sterne [...]. Aus einer anderen Perspektive könnte die Cyborgwelt gelebte soziale und körperliche Wirklichkeiten bedeuten, in der niemand mehr seine Verbundenheit und Nähe zu Tieren und Menschen zu fürchten braucht und niemand mehr vor dauerhaft partiellen Identitäten und widersprüchlichen Positionen zurückschrecken muß. Der politische Kampf besteht darin, beide Blickwinkel zugleich einzunehmen, denn beide machen sowohl Herrschaftsverhältnisse als auch Möglichkeiten sichtbar, die aus der jeweils anderen Perspektive unvorstellbar sind. ${ }^{39}$

Ihr Essay »Situiertes Wissen « (»Situated Knowledges «) verficht in ähnlicher Weise »eine doppelte Vision «: Im Gegensatz zu einer Wissenschaft, die von einem "göttlichen Trick, alles von nirgendwo aus sehen zu können « Gebrauch macht, geht es dem Feminismus »um die Wissenschaften des multiplen Subjekts mit (mindestens) doppelter Vision « ${ }^{40}$ »Vision «, so argumentiert Haraway überraschend, »kann dazu dienen, binäre Oppositionen zu vermeiden«, vorausgesetzt man beharrt »auf der Körperlichkeit aller Vision ${ }^{41}{ }^{41}$ Diese stellt eine alternative Metapher zur Verfügung, um das in ihrem Artikel eingangs angesprochene Dilemma in Angriff zu nehmen, nämlich wie Feministinnen in ihrem Schwanken zwischen entgegengesetzten Positionen mit der Objektivität fertig werden:

In unseren Bemühungen, die rutschige Stange zu erklimmen, die zu einem brauchbaren Objektivitätsbegriff führen soll, haben ich und andere Feministinnen uns in den Objektivitätsdebatten abwechselnd oder sogar gleichzeitig an beide Enden der Dichotomie geklammert [...], die ich in diesem Essay als radikalen Konstruktivismus versus feministisch-kritischen Empirismus skizzierte. Klettern ist natürlich schwierig, wenn man sich, egal ob gleichzeitig oder abwechselnd, an zwei gegenüberliegenden Enden einer Stange festhält. Zeit also, die Metaphern zu wechseln. ${ }^{42}$

Wenn also die Vision, d. h. die doppelte Vision oder vielmehr: das Aspekt-Sehen, eine Metapher zur Verfügung stellt, um die eigene Spaltung zwischen den Alternativen von radikalem Konstruktivismus und kritischem Empirismus in Angriff zu nehmen, dann scheint die verkörperte Vision mit ihrem partiellen, situierten Wissen der Ort zu sein, wo die Gegensätze zu jener eigentümlichen Art der Nicht-Synthese eines Kippbilds zusammenkommen.

Mehr noch als Canguilhems regionale Epistemologie sind Haraways situierte Wissensformen im lebendigen Köper und seiner kontingenten Geschichte in einer bestimmten sozio-politischen Umwelt situ- 
iert. Sie beruft sich auf diese Wissensformen vor allem im Hinblick auf eine Lehre einer »feministischen Objektivität ", auf eine »Lehre verkörperter Objektivität [...], die paradoxen und kritisch-feministischen Wissenschaftsprojekten Raum böte «. ${ }^{43}$ Dabei geht es insbesondere um das Problem, "wie wir zugleich die grundlegende historische Kontingenz aller Wissensansprüche und Wissenssubjekte in Rechnung stellen [...] und einem nicht-sinnlosen Engagement für Darstellungen verpflichtet sein können, die einer >wirklichen< Welt die Treue halten, einer Welt, die teilweise miteinander geteilt werden kann $«{ }^{44}$ Dies ist nicht lediglich ein epistemologisches Problem, sondern auch eine Frage der Politik und des Begehrens oder vielmehr eines »notwendigerweise vielfältige[n] Begehren[s] «, über das Haraway schreibt: »Alle Bestandteile dieses Begehrens sind paradox und gefährlich, und ihre Kombination ist sowohl widersprüchlich als auch notwendig. « ${ }^{45}$ Wenn Haraway im Weiteren behauptet "Feministinnen brauchen keine Objektivitätslehre, die Transzendenz verspricht ${ }^{46}$ und dann die beiden Metaphern ins Feld führt - zum einen, eine Stange hinaufzuklettern und sich gleichzeitig an beiden Enden festzuhalten, zum anderen die verkörperte, doppelte Vision -, so möchte ich mit dem Kippbild eine weitere Metapher vorschlagen: Darin werden widersprüchliche Elemente in einem Paradox vereint, dessen Lösung nicht in einer höheren, transzendierenden Synthese, sondern auf einer materiellen Ebene, in den multiplen Betrachtungsweisen desselben Materials, zu suchen ist. Es kann sowohl verwendet werden, um ein Begehren nach Objektivität, das zwischen gleichermaßen unbefriedigenden, sich gegenseitig widersprechenden Optionen festsitzt, als auch um die Gegenstände zu beschreiben, die durch ein solches Begehren konstituiert werden: Gegenstände in der Art von situierten Wissensformen, die mit einem Universalwissen ohne Kontingenz und Geschichtlichkeit brechen und zugleich Kontinuitäten erlauben, die das Wissen mitteilbar und politisch machen; oder aber einzelne Organismen, die ihre Teile integrieren und determinieren und zugleich autonom hinsichtlich ihrer Umwelt sind; oder aber schließlich das Leben, in dem das Begehren nach beständiger mechanischer Determinierung, die Erkenntnis und Kontrolle erlaubt, einem Begehren nach Diskontinuität gegenübersteht - eine Bedingung sowohl für die spontane Emergenz von genuinem Wandel als auch für die Subjekte objektiven Wissens.

Aus dem Englischen von Konrad Petrovszky 


\section{A N M ERKU N GE N}

1 Georges Canguilhem, "Einleitung. Das Denken und das Lebendige ", in ders., Die Erkenntnis des Lebens, übers. v. Till Bardoux, Maria Muhle u. Francesca Raimondi (Berlin: August Verlag, 2009), S. 16.

2 Ebd.

3 Ebd., S. 22.

4 Ebd.

5 Ebd.

6 Georges Canguilhem, "Aspekte des Vitalismus", in ders., Die Erkenntnis, S. 149-81, hier S. 153.

7 Ebd., S. 153-54.

8 Siehe Immanuel Kant, Kritik der reinen Vernunft, hg. v. Wilhelm Weischedel, 2 Bde. (Frankfurt a.M.: Suhrkamp, 1974), »Vom transzendentalen Schein «, I, S. 308-11 (A293-98; B350-55) und "Die Antinomie der reinen Vernunft", II, S. 399-512 (A405-567; B432-595); sowie ders., Kritik der Urteilskraft, hg. v. Wilhelm Weischedel (Frankfurt a.M.: Suhrkamp, 1974), »Dialektik der teleologischen Urteilskraft", S. 334-71 (A308-59; B311-63). Zu Kants »unvermeidlichem ", "erlaubtem " und sogar »erwünschtem Schein " und dem davon ausgehenden prekären kritischen Potenzial, siehe Astrid Deuber-Mankowsky, Praktiken der Illusion. Kant, Nietzsche, Cohen, Benjamin bis Donna J. Haraway (Berlin: Vorwerk 8, 2007).

9 Auch gemischte Formen sind vorstellbar: Während sich im Falle des HasenEnten-Bildes beim wiederholten Wechsel der unterschiedlichen Sichtweisen prinzipiell nichts ändert, lässt sich in übertragenen Fällen annehmen, dass etwas aus der einen in die jeweils andere Perspektive importiert werden und so zu einer Transformation jeder Sichtweise führen kann, ohne notwendigerweise zu einer Annäherung der beiden zu führen. In diesem Sinne waren sowohl der Vitalismus als auch der Mechanismus im Laufe der Geschichte beträchtlichen Transformationen unterworfen, während deren scharfer Gegensatz weiterhin festgestellt werden kann. Vgl. Hilde Hein, »The Endurance of the Mechanism-Vitalism Controversy", Journal of the History of Biology, 5.1 (1972), S. 159-88.

10 Canguilhems Einführung in Die Erkenntnis des Lebens beispielsweise spielt sicherlich auf den Vitalismus an, wenn er schreibt: "Wenn sich Denken und Erkenntnis durch den Menschen ins Leben einschreiben, um es zu regeln, so muss man folglich zugestehen, dass dieses selbe Leben nicht die mechanische, blinde und dumme Kraft sein kann, die man sich gerne darunter vorstellt, wenn man es dem Denken gegenüberstellt (Canguilhem, »Einleitung «, S. 17). Er fährt jedoch nicht damit fort, die Existenz irgendeiner intelligenten Lebenskraft zu behaupten, sondern kritisiert eher einen vermessenen Anthropozentrismus, wonach der Mensch »empört darüber, selbst ein Lebewesen zu sein, sich für seinen eigenen Gebrauch die Idee eines getrennten Reiches zurechtschmiedet « (ebd.).

11 »Der Vitalismus wäre demnach einfach die Anerkennung des Lebens als ursprünglicher Ordnung der Erscheinungen und somit der Besonderheit des biologischen Wissens« (Georges Canguilhem, Die Herausbildung des Reflexbegriffs 
im 17. und 18. Jahrhundert, übers. v. Henning Schmidgen [München: Fink, 2008], S. 140-41).

12 Ebd., S. 5.

13 Canguilhem, »Aspekte", S. 158.

14 Michel Foucault, "Vorwort von Michel Foucault", in ders., Schriften in vier Bänden. Dits et Ecrits, hg. v. Daniel Defert u. François Ewald (Frankfurt a.M.: Suhrkamp, 2001-05), III: 1976-1979, übers. v. Michael Bischoff u. a. (2003), S. 551-67, hier S. 562.

15 Astrid Deuber-Mankowsky und Christoph F. E. Holzhey, »Vitalismus als kritischer Indikator: Der Beitrag der Kulturwissenschaften an der Bildung des Wissens vom Leben ", in Der Einsatz des Lebens. Lebenswissen, Medialisierung, Geschlecht, hg. v. Astrid Deuber-Mankowsky, Christoph F. E. Holzhey u. Anja Michaelsen (Berlin: b_books, 2009), S. 9-30, hier S. 12-13, mit Bezug auf Deuber-Mankowsky, Praktiken der Illusion, S. 271-345.

16 Monica Greco, »On the Vitality of Vitalism«, Theory, Culture \& Society, 22.1 (2005), S. 15-27, hier S. 19.

17 Ebd.

18 Donna J. Haraway, Crystals, Fabrics, and Fields. Metaphors That Shape Embryos (Berkeley: North Atlantic Books, 2004), S. xix.

19 Ebd.

20 Ebd., S. 34.

21 Ebd., S. 2.

22 Ebd., S. 197 u. 199.

23 Ebd., S. xix.

24 Foucault, »Vorwort«, S. 562.

25 Canguilhem, Die Herausbildung des Reflexbegriffs, S. 208.

26 Canguilhem, »Aspekte «, S. 171.

27 Ebd., S. 173.

28 Ebd., S. 180. Ebenso ist er der Auffassung, dass eine marxistische Dialektik, die die Rückkehr des Vitalismus im frühen 20. Jahrhundert »mit den Vertrauenskrisen der bürgerlichen Gesellschaft bezüglich der Wirksamkeit der kapitalistischen Institutionen « in Verbindung bringt, nicht weit genug geht. "Die Dialektik ist in der Biologie vertretbar, weil es im Leben das gibt, was den Vitalismus, eher in Form eines Anspruchs als einer Doktrin, hervorgerufen hat und was seine Vitalität, sprich seine ihm eigene Spontaneität erklärt - was Claude Bernard zum Ausdruck brachte, als er sagte: >Leben ist Schöpfung « (Ebd., S. 179-80).

29 Georges Canguilhem, "Der Gegenstand der Wissenschaftsgeschichte «, in ders.: Wissenschaftsgeschichte und Epistemologie. Gesammelte Aufsätze, hg. v. Wolf Lepenies, übers. v. Michael Bischoff u. Walter Seiter (Frankfurt a.M.: Suhrkamp, 1979), S. 22-38, hier S. 33. Siehe hierzu Hans-Jörg Rheinberger, »Ein erneuter Blick auf die historische Epistemologie von Georges Canguilhem ", in Maß und Eigensinn. Studien im Anschluß an Georges Canguilhem, hg. v. Cornelius Borck, Henning Schmidgen u. Volker Hess (München: Fink, 2005), S. 223-27, hier S. 230.

30 Die Übersetzung weicht hier von der Standardübersetzung ab, die an dieser Stelle 
fehlerhaft ist. Vgl. Canguilhem, »Der Gegenstand «, S. 34. und ders., »L'objet de l'histoire des sciences", in ders., Études d'histoire et de philosophie des sciences, 7. erw. Aufl. (Paris: Vrin, 1994), S. 9-23, hier S. 21; Anm. d. Übers.

31 Ebd. Die deutsche Standardübersetzung ist an dieser Stelle unvollständig; vgl. die vorangehende Anmerkung.

32 Georges Canguilhem, »Die Zelltheorie«, in ders., Die Erkenntnis, S. 75-145, hier S. 78.

33 Ebd., S. 142.

34 Ebd., S. 143.

35 Ebd., S. 144-45.

36 Haraway, Crystals, S. xix.

37 Siehe dazu auch die Beiträge von Marie-Luise Angerer und Henning Schmidgen in diesem Band.

38 Donna J. Haraway, »Ein Manifest für Cyborgs. Feminismus im Streit mit den Technowissenschaften ", in dies., Die Neuerfindung der Natur. Primaten, Cyborgs und die Frauen, hg. v. Carmen Hammer u. Immanuel Stieß (Frankfurt a.M.: Campus, 1995), S. 33-72, hier S. 39.

39 Ebd., S. 40.

40 Donna J. Haraway, "Situiertes Wissen. Die Wissenschaftsfrage im Feminismus und das Privileg einer partialen Perspektive", in dies., Die Neuerfindung, S. 73-97, hier S. 81 u. 90.

41 Ebd., S. 80.

42 Ebd. Übers. modifiziert durch den Autor (C. H.).

43 Ebd.

44 Ebd., S. 78.

45 Ebd., S, 79.

46 Ebd. 
Christoph F. E. Holzhey, »Kippbilder des Vitalen: Lebenswissen bei Canguilhem und Haraway«, in Situiertes Wissen und regionale Epistemologie: Zur Aktualität Georges Canguilhems und Donna J.

Haraways, hg. v. Astrid Deuber-Mankowsky und Christoph F. E. Holzhey, Cultural Inquiry, 7 (Wien: Turia + Kant, 2013), S. 12139 <https://doi.org/10.37050/ci-07_06>

\section{REFERENCES}

Borck, Cornelius, Henning Schmidgen u. Volker Hess (Hg.), Maß und Eigensinn. Studien im Anschlu $\beta$ an Georges Canguilhem (München: Fink, 2005)

Canguilhem, Georges, Die Erkenntnis des Lebens, übers. v. Till Bardoux, Maria Muhle u. Francesca Raimondi (Berlin: August, 2009)

D Die Herausbildung des Reflexbegriffes im 17. und 18. Jahrhundert, übers. u. mit einem Vorwort eingel. v. Henning Schmidgen (München: Fink, 2008) <https://doi.org/10.30965/ 9783846745250>

Wissenschaftsgeschichte und Epistemologie. Gesammelte Aufsätze, hg. v. Wolf Lepenies, übers. v. Michael Bischoff u. Walter Seiter (Frankfurt a.M.: Suhrkamp, 1979)

Études d'histoire et de philosophie des sciences, 7. erw. Aufl. (Paris: Vrin, 1994)

Deuber-Mankowsky, Astrid, Praktiken der Illusion. Kant, Nietzsche, Cohen, Benjamin bis Donna J. Haraway (Berlin: Vorwerk 8, 2007)

Deuber-Mankowsky, Astrid u. Christoph F. E. Holzhey, »Vitalismus als kritischer Indikator: Der Beitrag der Kulturwissenschaften an der Bildung des Wissens vom Leben $\ll$, in Der Einsatz des Lebens. Lebenswissen, Medialisierung, Geschlecht, hg. v. Astrid DeuberMankowsky, Christoph F. E. Holzhey u. Anja Michaelsen (Berlin: b_books, 2009), S. 9-30

Foucault, Michel, Schriften in vier Bänden. Dits et Ecrits, hg. v. Daniel Defert u. François Ewald (Frankfurt a.M.: Suhrkamp, 2001-05)

Greco, Monica, »On the Vitality of Vitalism《, Theory, Culture \& Society, 22.1 (2005), S. 15-27 <https://doi.org/10.1177/0263276405048432>

Haraway, Donna J., Crystals, Fabrics, and Fields. Metaphors That Shape Embryos (Berkeley, CA: North Atlantic Books, 2004)

-Die Neuerfindung der Natur. Primaten, Cyborgs und Frauen, hg. u. eingel. v. Carmen Hammer u. Immanuel Stieß (Frankfurt a.M.: Campus, 1995)

Hein, Hilde, $\gg$ The Endurance of the Mechanism-Vitalism Controversy «, Journal of the History of Biology, 5.1 (1972), S. 159-88 <https://doi.org/10.1007/BF02113490>

Kant, Immanuel, Kritik der reinen Vernunft, hg. v. Wilhelm Weischedel, 2 Bde. (Frankfurt a.M.: Suhrkamp, 1974)

Kritik der Urteilskraft, hg. v. Wilhelm Weischedel (Frankfurt a.M.: Suhrkamp, 1974) 